\title{
ON BENNETT'S CONJECTURE AND COMPLETE MONOTONICITY
}

\section{ULRICH ABEL AND VitALIY KUSHNIREVYCH}

Abstract. Bennett [1] gave a generalization of Schur's theorem in order to study various momentpreserving transformations. Recently, Su [5] confirmed a monotonicity conjecture of Bennett which is related to the generalized Schur's theorem and Haber's inequality. In this paper we present a short proof of this result which is based on a combinatorial identity. Moreover, we show that the function in Bennett's conjecture is not only monotonically decreasing but completely monotonic. Furthermore, we give its explicit representation as a Laplace integral which implies the complete monotonicity. Finally, we prove a multivariate version of the above-mentioned combinatorial identity.

Mathematics subject classification (2010): 26A06, 44A10, 05A19.

Keywords and phrases: Complete monotonicity, representation as a Laplace integral.

\section{REFERENCES}

[1] G. Bennett, Hausdorff means and moment sequences, Positivity 15 (2011), 17-48.

[2] G. H. Hardy, J. E. Littlewood, G. Pólya, Inequalities, Cambridge University Press, Cambridge, 1952.

[3] H. Y. L. LiAng, L. Mu, Y. WAnG, Catalan-like numbers and Stieltjes moment sequences, Discrete Math. 339 (2016), 484-488.

[4] J. A. Shohat, J. D. Tamarkin, The Problem of Moments, Amer. Math. Soc., New York, 1943.

[5] X.-T. Su, Proof of a monotonicity conjecture, Math. Inequal. Appl. 21 (2018), 91-98.

[6] Y. WAng, B.-X. ZHU, Log-convex and Stieltjes moment sequences, Adv. in Appl. Math. 81 (2016), $115-127$

[7] D. V. WIDDER, The Laplace Transform, Princeton University Press, Princeton, 1946. 